\title{
Supernova Remnant CTB 80 and PSR 1951+32
}

\author{
R. G. Strom ${ }^{1,2}$ and B. W. Stappers ${ }^{2}$ \\ ${ }^{1}$ NFRA, Radiosterrenwacht, Postbus 2, Dwingeloo, Netherlands \\ ${ }^{2}$ Astronomical Institute, University of Amsterdam, Netherlands
}

\begin{abstract}
.
The $39.5 \mathrm{~ms}$ pulsar PSR $1951+32$ and the remnant with which it is associated, CTB 80 , have both been observed with the recently upgraded Westerbork synthesis array (WSRT). Parts of CTB 80 have been observed over the frequency coverage from 0.3 to $8 \mathrm{GHz}$, while PSR $1951+32$ has been observed from 0.3 to $1.6 \mathrm{GHz}$. The results are described and discussed in the context of the pulsar-remnant association.
\end{abstract}

"Whence and what art thou, execrable shape?" John Milton, Paradise Lost

\section{Introduction}

The extended nonthermal galactic radio source CTB 80 has been the subject of high resolution mapping since 1980 (Strom, Angerhofer, \& Velusamy 1980; Angerhofer et al. 1981). Its unusual morphology, and distinct components, were evident from the beginning. Although sometimes classified as a composite supernova remnant (SNR), the fact is that there is little sign of shell structure in the extended emission, where several arcs of emission emanate from a central plateau (Fig. 1). Indeed, Green (1991), in his catalogue of remnants, asks "Is it a SNR?".

X-ray emission has been imaged from the central region of CTB 80 (SafiHarb, Ögelman, \& Finley 1995), both the flat spectrum core and the extended plateau to the east. Optical emission from the core (Strom et al. 1980) has been imaged and studied spectroscopically (Hester \& Kulkarni 1989; Greidanus \& Strom 1990). There is extended infrared emission seen along the arc to the northwest, which bends around to the south, forming a partial ring (Fesen, Shull, \& Saken 1988).

\section{CTB 80 - a peculiar supernova remnant}

The large scale structure of CTB 80 consists of several arm-like features emanating from the central core (Fig. 1). The core has a flat spectrum $(\alpha \simeq 0)$, and although the extended emission to the east has been said to exhibit a steepening spectral gradient (Angerhofer et al. 1981), a comparison of 49 and $92 \mathrm{~cm}$ Westerbork maps shows no significant trend. At short wavelengths and high resolution, the flat spectrum core is limb brightened along its western and southern edges 


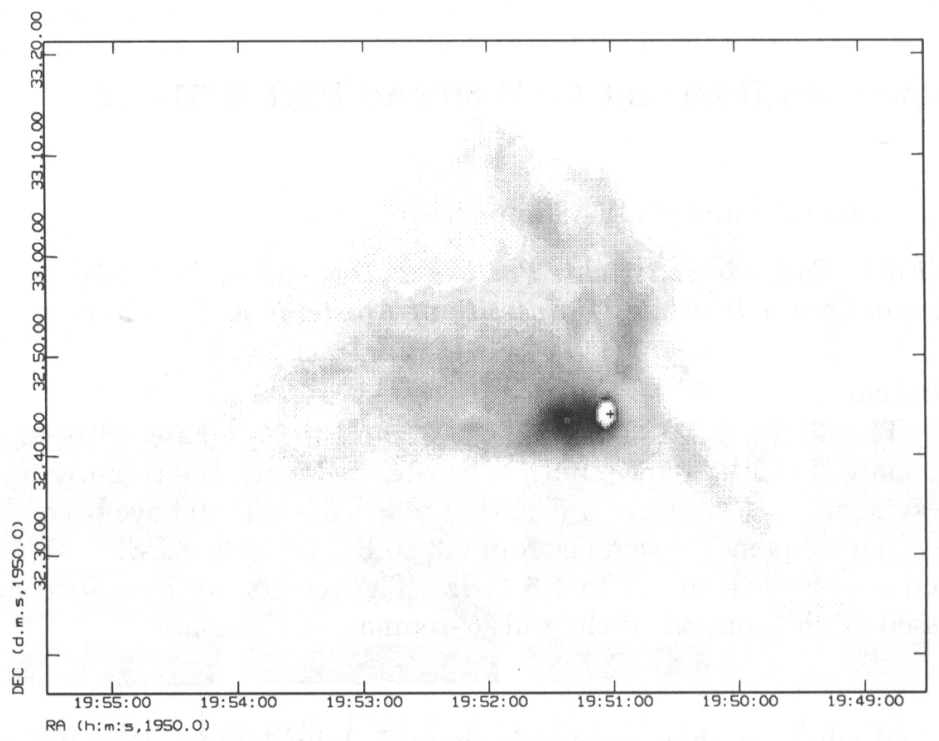

Figure 1. A $92 \mathrm{~cm}$ map of CTB 80 made with the WSRT at a resolution of $52^{\prime \prime} \times 100^{\prime \prime}$. A cross marks the location of the pulsar PSR $1951+32$ at the edge of the flat spectrum core (white ellipse).

(Fig. 2). HI absorption against this feature extends to a velocity of $-16 \mathrm{~km} \mathrm{~s}^{-1}$ (Fig. 3) which is consistent with a distance of around $2 \mathrm{kpc}$.

\subsection{PSR 1951+32 - the neutron star}

A pulsar was discovered near the edge of the flat spectrum core, first as a steep spectrum, polarized point source (Strom 1987), and subsequently through its $39.5 \mathrm{~ms}$ pulsed emission (Kulkarni et al. 1988). The pulsar is remarkable not only for its short period, $P$, but also for its low $P / \dot{P}$, leading to a low inferred magnetic field strength. This was in some respects not too surprising, however, given the luminosity of the core component: the combination of $P$ and $\dot{P}$ have to provide an $\dot{E}$ not much in excess of $10^{34} \mathrm{ergs} \mathrm{s}^{-1}$.

We have made observations of PSR $1951+32$ with PuMa and the WSRT at $21 \mathrm{~cm}$. The pulse shape is similar to what has been observed in the past (Kulkarni et al. 1988), with clear evidence of linear polarization. From the change in position angle over our band, we estimate that the rotation measure $(R M)$ seen is similar to that found for the extended emission in CTB 80.

\section{Discussion}

What are we to make of this perplexing source, with its unusual morphology and disparate components? Let us first consider whether the components pulsar, flat spectrum core, extended emission - all belong together. It has been 


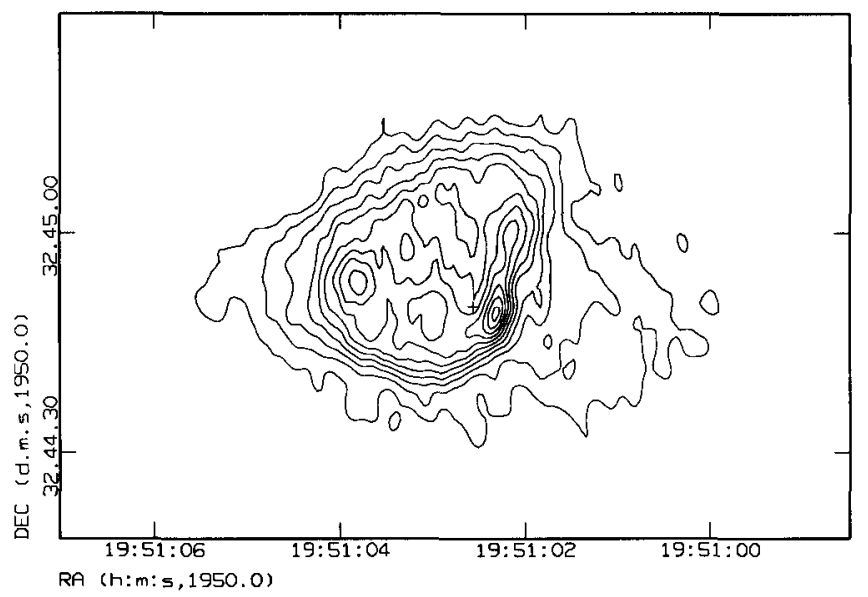

Figure 2. A $3.6 \mathrm{~cm}$ map of the flat-spectrum core of CTB 80 made with the WSRT at a resolution of $2{ }^{\prime \prime} 1 \times 33^{\prime \prime} 9$. The pulsar, located just to the east of the brightest peak, is indicated by a cross

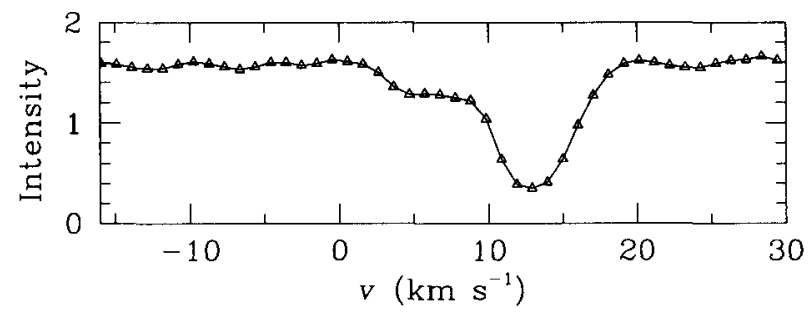

Figure 3. HI absorption against the flat-spectrum core of CTB 80

argued that the pulsar/neutron star powers the core (Strom 1987), and indeed that it is an example of a pulsar wind nebula (Hester \& Kulkarni 1989). The fact that the core has a flat spectrum à la Crab Nebula also suggests an association. Moreover, the pulsar's $D M$ and the distance indicated by HI absorption are consistent. The association between the pulsar/core and the extended emission is less secure, though their similar $R M \mathrm{~s}$ are at least consistent with it.

A number of timescales can be determined on the basis of various physical arguments. The spindown time of PSR 1951+32, the expansion "age" of an HI shell surrounding the large scale emission, and very crude estimates of the age of the outer arms for "typical" remnant expansion rates, give roughly consistent values near $10^{5} \mathrm{yr}$ (Table 1 ). The time required for the pulsar to have moved from the central area of the extended emission to its present location is somewhat less than this but, given all the uncertainties, probably not impossibly discordant. The expansion age of the core based upon the kinematics of its $\mathrm{H} \alpha$ emission is nearly two orders of magnitude below the other indicators, but it probably has more to do with recent excitation than the total time it has been underway. 
Finally, the spectral break in the radio emission from the core (at $\nu \simeq 80 \mathrm{GHz}$, Salter et al. 1989), if interpreted as due to synchrotron losses, implies an age 100 times larger than the rest if the average magnetic field strength has been near its present equipartition value. (Consistency would require $B_{\mathrm{av}} \simeq 10 B_{\mathrm{eq}}$.)

Table 1. CTB 80 and PSR 1951+32: timescales

Indicator

$P / 2 \dot{P}$

$r / v_{\mathrm{PSR}}$

HI shell $\left(r / v_{\mathrm{HI}}\right)$

SNR (assuming $v_{\mathrm{av}}=200-1000 \mathrm{~km} \mathrm{~s}^{-1}$ )

Core expansion (kinematics of $\mathrm{H} \alpha$ )

Core spectral break (assuming $B=B_{\mathrm{eq}}$ )
Timescale (yr)

107000

38000

77000

$100000-10000$

1500

10000000

The timescales summarized in Table 1 point to the explosion of a massive star some 100000 years ago which produced a fast moving and still active pulsar. The supernova shock waves have excited the extended (nonthermal, HI, infrared and X-ray) emission. (It has been suggested by Fesen et al. (1988) that the neutron star has recently overtaken the decelerating shell, and is now re-exciting it.) While this scenario may be roughly correct, many loose ends still need to be tidied up.

Acknowledgments. The Westerbork Observatory is operated by the Netherlands Foundation for Research in Astronomy with financial support from the Netherlands Organisation for Scientific Research (NWO).

\section{References}

Angerhofer, P. E., Strom, R. G., Velusamy, T., Kundu, M. R. 1981, A\&A, 94, 313

Fesen, R. A., Shull, J. M., Saken, J. M. 1988, Nat, 334, 229

Green, D. A. 1991, PASP, 103, 209

Greidanus, H., Strom, R. G. 1990, A\&A, 240, 376

Hester, J. J., Kulkarni, S. R. 1989, ApJ, 340, 362

Kulkarni, S. R., Clifton, T. C., Backer, D. C., Foster, R. S., Fruchter, A. S., Taylor, J. H. 1988, Nat, 331, 50

Safi-Harb, S., Ögelman, H., Finley, J.P. 1995, ApJ, 439, 722

Salter, C. J., Reynolds, S. P., Hogg, D. E., Payne, J. M., Rhodes, P. J. 1989, ApJ, 338, 171

Strom, R. G. 1987, ApJ, 319, L103

Strom, R. G., Angerhofer, P. E., Velusamy, T. 1980, Nat, 284, 38 